\title{
Even Small Decreases in Blood Pressure during Conscious Sedation Affect Clinical Outcome after Stroke Thrombectomy: An Analysis of Hemodynamic Thresholds
}

\author{
(D) M.K. Whalin, (D) K.M. Halenda, (DD.C. Haussen, DL.C. Rebello, DM.R. Frankel, DR.Y. Gershon, and (D) R.G. Nogueira
}

\begin{abstract}
BACKGROUND AND PURPOSE: The adverse effects of general anesthesia in stroke thrombectomy have been attributed to intraprocedural hypotension, yet optimal hemodynamic targets remain elusive. Identifying hemodynamic thresholds from patients without exposure to general anesthesia may help separate the effect of hypotension from the effect of anesthesia in thrombectomy outcomes. Therefore, we investigated which hemodynamic parameters and targets best correlate with outcome in patients treated under sedation with monitored anesthesia care.
\end{abstract}

MATERIALS AND METHODS: We performed a retrospective analysis of a prospectively collected data base of patients with anterior circulation stroke who were successfully reperfused (modified $\mathrm{TICI} \geq 2 \mathrm{~b}$ ) under monitored anesthesia care sedation from 2010 to 2015. Receiver operating characteristic curves were generated for the lowest mean arterial pressure before reperfusion, both as absolute values and relative changes from baseline. Cutoffs were tested in binary logistic regression models of poor outcome (90-day mRS $>2$ ).

RESULTS: Two-hundred fifty-six of 714 patients met the inclusion criteria. In a multivariable model, a $\geq 10 \%$ mean arterial pressure decrease from baseline had an OR for poor outcome of $4.38(95 \% \mathrm{Cl}, 1.53-12.56 ; P<.01)$. Other models revealed that any mean pressure of $<85 \mathrm{~mm}$ $\mathrm{Hg}$ before reperfusion had an OR for poor outcome of $2.22(95 \% \mathrm{Cl}, 1.09-4.55 ; P=.03)$ and that every $10-\mathrm{mm} \mathrm{Hg}$ drop in mean arterial pressure below $100 \mathrm{~mm} \mathrm{Hg}$ had an OR of $1.28(95 \% \mathrm{Cl}, 1.01-1.62 ; P=.04)$.

CONCLUSIONS: $A \geq 10 \%$ mean arterial pressure drop from baseline is a strong risk factor for poor outcome in a homogeneous population of patients with stroke undergoing thrombectomy under sedation. This threshold could guide hemodynamic management of patients during sedation and general anesthesia.

ABBREVIATIONS: $M A C=$ monitored anesthesia care; $M A P=$ mean arterial pressure

$\mathbf{R}$ etrospective studies have found that performing thrombectomies with the patient under general anesthesia may be associated with worse outcomes, ${ }^{1,2}$ possibly because of the increased incidence and severity of hypotension compared with the use of conscious sedation. ${ }^{3}$ Hypotension before reperfusion may compromise collateral blood flow and negatively impact outcomes in acute ischemic stroke. Previous investigations demonstrated that a lowest recorded systolic blood pressure of $>140 \mathrm{~mm} \mathrm{Hg}$ during endovascular therapy for acute ischemic stroke is associated with good neurologic outcome. ${ }^{3}$ Our group subsequently showed that a lowest mean arterial pressure (MAP) of $\geq 70 \mathrm{~mm} \mathrm{Hg}$ is an independent predictor of favorable neurologic outcome. ${ }^{4}$

Received May 11, 2016; accepted after revision September 6.

From the Departments of Anesthesiology (M.K.W., R.Y.G.) and Neurology (D.C.H., L.C.R., M.R.F., R.G.N.) and Discovery Program (K.M.H.), Emory University School of Medicine, Atlanta, Georgia; and the Marcus Stroke and Neuroscience Center (D.C.H., L.C.R., M.R.F., R.G.N.), Grady Memorial Hospital, Atlanta, Georgia.

Please address correspondence to Raul G. Nogueira, MD, 49 Jesse Hill Jr Drive SE, Room 333, Atlanta, GA 30303; e-mail: raul.g.nogueira@emory.edu

http://dx.doi.org/10.3174/ajnr.A4992
These studies were limited because they included a mix of patients receiving conscious sedation and general anesthesia. In this setting, the association between general anesthesia and hypotension may make it difficult to discern the independent effects of blood pressure regardless of anesthesia type. We hypothesized that hypotension influences outcome even in those patients not exposed to general anesthesia. Our aim was to determine optimal hemodynamic parameters and thresholds for patients with acute stroke undergoing thrombectomy under conscious sedation with monitored anesthesia care (MAC). Such parameters could be used in future studies of outcome and anesthesia type to determine whether general anesthesia has deleterious effects independent of hypotension.

\section{MATERIALS AND METHODS}

After approval from the institutional review board, we retrospectively reviewed the records of 714 patients who underwent endovascular treatment for acute ischemic stroke in the neurointerventional suite of Grady Memorial Hospital from September 2010 to April 2015. Five hundred ninety-nine patients had anterior circulation stroke with 90 -day outcome data. We had complete 
hemodynamic data for 309 of the 347 patients treated under MAC. Two-hundred seventy-six of these patients were successfully reperfused (as defined by modified Thrombolysis in Cerebral Infarction $2 \mathrm{~b} / 3),{ }^{5}$ with a median procedure time of 64 minutes (interquartile range, 43-96 minutes). We excluded 20 patients $(7 \%)$ who were not reperfused within 2.5 hours of groin puncture, which left a study population of 256 . The baseline characteristics and periprocedural variables of our study population were collected prospectively within the study period (September 2010 to April 2015) from the Marcus Stroke and Neuroscience Center endovascular data base. Good outcome was defined as a 90-day modified Rankin Scale score of 0-2.

\section{Stroke Variables and Procedures}

Patients were selected for thrombectomy on the basis of the presence of salvageable tissue on imaging. TICI scores were determined in the angiography suite by the operator. Three-fourths of the procedures were performed with stent retrievers, and the remainder used thromboaspiration. All patients had follow-up imaging to assess hemorrhage. The modified Rankin Scale scores at 90 days were determined by an in-person follow-up appointment. Occasional no-shows were estimated by a structured phone interview. ${ }^{6}$

\section{Anesthesia Variables}

Our anesthesia care team includes an attending anesthesiologist supervising a nurse anesthetist or anesthesiologist assistant. More than $95 \%$ of our MAC procedures use dexmedetomidine infusions with supplemental fentanyl and midazolam as needed. For most patients with MAC, blood pressure measurements are noninvasive and arterial lines are usually reserved for patients with severe cardiac disease. Infusion regimens vary by practitioner but usually involve a loading dose of $0.5-1 \mu \mathrm{g} / \mathrm{kg}$ delivered during 10 minutes followed by a maintenance infusion of $0.5-1 \mu \mathrm{g} / \mathrm{kg} / \mathrm{hour}$. Although we did not have a formal protocol for hemodynamic management during this time, the target range for systolic blood pressure was usually set between 140 and $180 \mathrm{~mm} \mathrm{Hg}$ before reperfusion. Phenylephrine was the most commonly used vasopressor. Anesthetic variables, including type of sedation, use of vasopressors, and systolic and diastolic blood pressure values (charted every 5 minutes), were retrieved from the anesthesia records. The baseline systolic and diastolic pressures were determined by averaging the measurements before the start of sedation. The single lowest MAP before successful reperfusion was recorded with its component systolic and diastolic blood pressures. We focused on MAP variables because mean blood pressure is directly measured by the noninvasive cuffs used on most patients, whereas these cuffs use an algorithm to derive systolic and diastolic blood pressure. $^{7}$

\section{Statistical Analysis}

All data are expressed as mean \pm SD or median with interquartile range as appropriate. Receiver operating characteristic curves were generated to identify hemodynamic cut-points. Between groups, 2-sided comparisons for continuous/ordinal variables were made with the Student $t$ test or Mann-Whitney $U$ test as appropriate. Categoric variables were compared by $\chi^{2}$ or Fisher exact tests as appropriate. Variables with a $P<.10$ in these uni- variate analyses were simultaneously entered into multivariate regression models to identify which were independent predictors of outcome. We used binary logistic regression models with significance set as $P<.05$. Statistical analyses were performed by using SPSS Statistics, Version 23 (IBM,'Armonk, New York).

\section{RESULTS}

Two hundred fifty-six patients meeting the selection criteria were included in the analysis. The mean age of the study population was $65.2 \pm 15.4$ years, and the mean NIHSS score was 17.3 \pm 5.9 . Table 1 presents the clinical characteristics and procedural variables of the groups with good and poor outcomes at 90 days. The groups had similar procedure durations and times from last known healthy to groin puncture, as well as similar rates of IV tPA and stent retriever use. We observed differences in ASPECTS and NIHSS scores and rates of parenchymal hemorrhage between the groups. The poor outcome group also had higher rates of atrial fibrillation and ICA terminus occlusion.

Table 2 shows systolic blood pressure and MAP parameters in the population and their association with outcome. Patients included in the analysis had a mean baseline systolic blood pressure of $157.6 \pm 29.3 \mathrm{~mm} \mathrm{Hg}$ and a MAP of $108.5 \pm 18.6 \mathrm{~mm} \mathrm{Hg}$. The means for the lowest recorded systolic blood pressure and MAP before successful reperfusion were 118.0 \pm 22.0 and $80.7 \pm 14.4$ $\mathrm{mm} \mathrm{Hg}$, respectively. Baseline pressures did not differ between the 2 outcome groups, but all variables related to the lowest pressure before reperfusion were statistically different (Table 2).

Due to collinearity, we incorporated the continuous variables of the lowest MAP, absolute MAP drop, and percentage MAP drop one at a time into binary logistic regression models for predictors of poor outcome. Neither absolute MAP drop nor percentage MAP drop were independent predictors of outcome $(P=.38$ and .16 , respectively). The lowest MAP before modified TICI $2 \mathrm{~b} / 3$ reperfusion was independently associated with poor outcome $(P=.04)$. We performed a linear transformation [(100 - lowest MAP $) / 10]$ to place this result in a more meaningful clinical context. As shown in Table 3, the odds of poor outcome increased by $28 \%$ for every $10 \mathrm{~mm} \mathrm{Hg}$ that the lowest MAP fell below $100 \mathrm{~mm} \mathrm{Hg}$.

We next generated receiver operating characteristic curves to investigate hemodynamic thresholds that could predict neurologic outcome (Fig 1). The areas under the curve for the MAP variables were approximately 0.6 , suggesting that they are weaker predictors than the NIHSS score (0.74) or ASPECTS (0.65). For ease of comparison, we linked the pressures to the mRS category that generated curves above the reference line. For example, a lowest MAP of $>90$ $\mathrm{mm} \mathrm{Hg}$ was $>80 \%$ specific for good outcome (Fig $1 A$ ), whereas a drop in MAP of $>40 \mathrm{~mm} \mathrm{Hg}$ was approximately $80 \%$ specific for poor outcome (Fig $1 B$ ). The trade-off for the high specificities of these extreme values is low sensitivity. The $30 \%$ sensitivity of a lowest MAP of $>90 \mathrm{~mm} \mathrm{Hg}$ indicates that many patients whose MAP falls below $90 \mathrm{~mm} \mathrm{Hg}$ will still have a good outcome.

In multivariate analysis for predictors of poor outcome, the lowest MAP of $<85 \mathrm{~mm} \mathrm{Hg}$ had an OR of 2.23 (95\% CI, 1.09$17.7 ; P=.03)$. The lowest MAP cutoffs of 70 and $80 \mathrm{~mm} \mathrm{Hg}$ were also independent predictors $(\mathrm{OR}=2.15 ; 95 \% \mathrm{CI}, 1.02-4.56$; $P=$ .04 ; and $\mathrm{OR}=2.21 ; 95 \% \mathrm{CI}, 1.12-4.16 ; P=.02$, respectively). For blood pressure changes, an absolute MAP drop of $>15 \mathrm{~mm} \mathrm{Hg}$ 
Table 1: Univariate analysis of baseline characteristics, procedural variables, and outcomes associated with 90-day $\mathrm{mRS}>2^{\mathrm{a}}$

\begin{tabular}{|c|c|c|c|c|}
\hline & $\begin{array}{l}\text { All Patients } \\
\left(n=256^{\mathrm{b}}\right)\end{array}$ & $\begin{array}{c}\text { 90-Day mRS 0-2 } \\
(n=152)\end{array}$ & $\begin{array}{c}90-\text { Day } m R S>2 \\
(n=104)\end{array}$ & $\begin{array}{c}P \\
\text { Value }\end{array}$ \\
\hline \multicolumn{5}{|l|}{ Demographics } \\
\hline Age (yr) & $65.2 \pm 15.4$ & $61.5 \pm 15.1$ & $70.7 \pm 14.1$ & $<.01^{\mathrm{c}}$ \\
\hline Male sex & $123(48 \%)$ & $76(50 \%)$ & $47(46 \%)$ & .49 \\
\hline Current smoker & 48 (19\%) & $30(20 \%)$ & 18 (17\%) & .61 \\
\hline Hypertension & $179(70 \%)$ & $102(67 \%)$ & $77(74 \%)$ & .23 \\
\hline Diabetes mellitus & $58(23 \%)$ & $35(23 \%)$ & $23(22 \%)$ & .86 \\
\hline Dyslipidemia & $92(36 \%)$ & $55(36 \%)$ & $37(36 \%)$ & .92 \\
\hline Atrial fibrillation & $95(37 \%)$ & $49(32 \%)$ & $46(44 \%)$ & $.06^{c}$ \\
\hline \multicolumn{5}{|l|}{ Stroke features } \\
\hline Baseline NIHSS & $17(13-22)$ & 15 (11-19) & $20(17-24)$ & $<.01^{\mathrm{c}}$ \\
\hline ASPECTS & $8(7-9)$ & $9(7-9)$ & $7(6-9)$ & $<.01^{\mathrm{c}}$ \\
\hline \multicolumn{5}{|l|}{ Occlusion site } \\
\hline ICA terminus & $46(18 \%)$ & $18(12 \%)$ & $28(27 \%)$ & $<.01^{\mathrm{c}}$ \\
\hline MCA MI & $158(72 \%)$ & $98(64 \%)$ & $60(58 \%)$ & .27 \\
\hline Last healthy to puncture (min) & $295(219-457)$ & $281(208-460)$ & $305(236-449)$ & .34 \\
\hline IV tPA given & $113(44 \%)$ & $71(47 \%)$ & $42(41 \%)$ & .35 \\
\hline \multicolumn{5}{|l|}{ Intraprocedural management } \\
\hline Stent retriever & $189(74 \%)$ & $115(76 \%)$ & $74(72 \%)$ & .44 \\
\hline Procedure time (min) & $62(41-88)$ & $60(41-81)$ & 66 (40-91) & .23 \\
\hline Vasopressor use & $134(52 \%)$ & $75(49 \%)$ & $59(57 \%)$ & .24 \\
\hline \multicolumn{5}{|l|}{ Outcomes } \\
\hline Parenchymal hemorrhage & $19(7 \%)$ & $4(3 \%)$ & $15(14 \%)$ & $<.01^{\mathrm{c}}$ \\
\hline Final infarct volume $\left(\mathrm{cm}^{3}\right)$ & $20.5(7.3-60.0)$ & $13.4(5.4-35.6)$ & 30.6 (17.4-111) & $<.01$ \\
\hline 90-day mRS & $2(1-4)$ & & & \\
\hline Mortality & $31(12 \%)$ & & $31(30 \%)$ & \\
\hline
\end{tabular}

Note:-M1 indicates the sphenoidal segment of middle cerebral artery.

${ }^{a}$ Results are shown as mean $\pm S D$, median (interquartile range), or number (percentage).

${ }^{\mathrm{b}}$ Not all patients had data available for ASPECTS $(n=243)$, minutes from last known healthy to groin puncture $(n=211)$,

or final infarct volume $(n=217)$.

' $V$ ariables with $P<.1$ added to the multivariate models.

Table 2: Intraprocedural hemodynamic characteristics ${ }^{a}$ and their association with 90-day mRS $>2$

\begin{tabular}{|c|c|c|c|c|}
\hline & $\begin{array}{l}\text { All Patients } \\
(n=256)\end{array}$ & $\begin{array}{l}\text { 90-Day mRS 0-2 } \\
(n=152)\end{array}$ & $\begin{array}{c}90-\text { Day } m R S>2 \\
(n=104)\end{array}$ & $\begin{array}{c}P \\
\text { Value }^{b}\end{array}$ \\
\hline \multicolumn{5}{|l|}{ Systolic blood pressure } \\
\hline Baseline (mm Hg) & 158 (135-176) & $156(134-173)$ & 160 (137-180) & .137 \\
\hline $\begin{array}{l}\text { Lowest before } \mathrm{mTICI} 2 \mathrm{~b} / 3 \\
(\mathrm{~mm} \mathrm{Hg})^{c}\end{array}$ & $117(103-132)$ & 118 (105-135) & 115 (99-124) & .047 \\
\hline Absolute drop $(\mathrm{mm} \mathrm{Hg})^{\mathrm{d}}$ & $35.5(17-56)$ & $30.0(11-51)$ & $41.5(24-63)$ & .004 \\
\hline$\%$ Pressure drop & $22.8 \%(12-34)$ & $21.4 \%(9-32)$ & $27.6 \%(16-37)$ & .002 \\
\hline \multicolumn{5}{|l|}{ Mean arterial pressure } \\
\hline Baseline (mm Hg) & $107(95-120)$ & $107(94-118)$ & $107(95-123)$ & .473 \\
\hline $\begin{array}{l}\text { Lowest before } \mathrm{mTICl} 2 \mathrm{~b} / 3 \\
(\mathrm{~mm} \mathrm{Hg})\end{array}$ & 79 (71-89) & $81(72-92)$ & $77(68-85)$ & .01 \\
\hline Absolute drop $(\mathrm{mm} \mathrm{Hg})^{\mathrm{d}}$ & 24.7 (13-39) & $23.8(11-31)$ & $26.7(16-42)$ & .008 \\
\hline$\%$ Pressure drop ${ }^{\mathrm{e}}$ & $23.3 \%(13-34)$ & $22.1 \%(12-31)$ & $25.6 \%(18-37)$ & .004 \\
\hline
\end{tabular}

Note:- $\mathrm{mTICl}$ indicates modified TICI

${ }^{\text {a }}$ Reported as median (interquartile range) unless otherwise noted.

${ }^{\mathrm{b}}$ Mann-Whitney $U$ test.

${ }^{c}$ Obtained from the systolic component of the lowest observed MAP prior to $\mathrm{mTICI} 2 \mathrm{~b} / 3$ reperfusion.

${ }^{\mathrm{d}}$ Calculated as the baseline pressure minus the lowest pressure before $\mathrm{mTICI} 2 \mathrm{~b} / 3$.

${ }^{e}$ Calculated as the absolute pressure drop divided by the baseline pressure.

was independently associated with poor outcome $(\mathrm{OR}=2.33$; 95\% CI, 1.11-18.6; $P=.03$ ). Finally, the largest OR for poor outcome was 4.38 for a MAP drop of $>10 \%$ (95\% CI, 1.53-12.6; $P<.01)$.

Because the lowest MAP predicted an outcome over a range of cutoffs, we next examined how its effect varied by the baseline NIHSS score. Given our sample size, we divided our study population into 3 groups by baseline NIHSS. Subdividing these by the lowest MAP yielded subgroups of 18-26 patients. The rates of good outcome are shown in Fig 2. For less severe strokes (NIHSS score, <15), even patients whose MAP fell below 70 $\mathrm{mm} \mathrm{Hg}$ had a $78 \%$ rate of good outcome. Only $60 \%$ of patients with NIHSS scores of $\geq 15$ had good outcomes, even when their MAPs stayed above $90 \mathrm{~mm}$ Hg.

\section{DISCUSSION}

The optimal hemodynamic management of patients undergoing endovascular therapy for acute ischemic stroke remains a topic of debate among clinicians. The 2014 Society for Neuroscience in Anesthesiology and Critical Care Expert Consensus Statement recommends the use of continuous hemodynamic monitoring with maintenance of systolic blood pressure at $140-180 \mathrm{~mm}$ $\mathrm{Hg},{ }^{8}$ but these recommendations are not based on data from patients having undergone thrombectomy. Instead, they originate from large epidemiologic studies that examined the associations between poor outcome and baseline hemodynamics or variation during the acute phase of acute ischemic stroke. ${ }^{9-11}$ Davis et $\mathrm{al}^{3}$ have investigated the association between favorable neurologic outcome and the lowest systolic blood pressure of $>140 \mathrm{~mm} \mathrm{Hg}$ based on the same extrapolation. In a subsequent study, our group selected a threshold of MAP of $>70 \mathrm{~mm} \mathrm{Hg}$ after dividing our range of observed MAP into quartiles. ${ }^{4}$ Most recently, Löwhagen Hendén et $\mathrm{al}^{12}$ showed that a $>40 \%$ fall in MAP during endovascular reperfusion with the patient under general anesthesia is an independent predictor of poor neurologic outcome. We saw a need to more closely examine how various blood pressure changes influence outcome for patients undergoing thrombectomy.

We sought to perform our analysis in a large but relatively homogeneous study population to best observe the relationship between intraprocedural hypotension and outcome. We chose to restrict our analysis to those patients who achieved successful reperfusion (modified TICI $2 \mathrm{~b} / 3$ ) because even the best blood pressure control would be unlikely to improve outcome if the vessel remained occluded. Because hypotension has been shown to be collinear with general anesthesia, ${ }^{3}$ we restricted our analysis to those patients whose procedures were performed under MAC to eliminate confounding by anesthesia type.

Despite the perceived hemodynamic stability of thrombec- 
tomy with the patient under sedation, we observed substantial drops in blood pressure during the procedures. Our baseline and lowest pressures are similar to the MAC/sedation cohorts reported from the Cleveland Clinic $(n=99)^{13}$ and Mayo Clinic $(n=38) .{ }^{14}$ Rates of vasopressor use were also quite similar across the 3 studies. The results from these 3 institutions suggest that it is difficult to replicate the tight hemodynamic control in the Calgary cohort reported by Davis et $\mathrm{al}^{3}$ One potential reason might be differences in the depth of sedation or drugs used. Although the former is impossible to determine, we can make crude comparisons of the latter. The Calgary patients received only fentanyl and midazolam for sedation, whereas our institution uses dexmedetomidine infusions with supplemental fentanyl and midazolam if needed. The practice at the Cleveland Clinic mirrors ours except they also use propofol infusions. ${ }^{13}$ In contrast, most patients at the Mayo Clinic received only fentanyl and midazolam for sedation but still experienced significant drops in blood pressure. ${ }^{14}$ It

Table 3: Binary logistic regression model for poor neurologic outcome (90-day mRS $>2)^{a}$

\begin{tabular}{lcc}
\multicolumn{1}{c}{ Variable } & OR (95\% CI) & $P$ Value \\
\hline Age & $1.05(1.02-1.07)$ & .001 \\
Atrial fibrillation & $1.09(0.55-2.15)$ & .805 \\
Baseline NIHSS & $1.16(1.09-1.24)$ & $<.001$ \\
ASPECTS & $0.80(0.65-0.97)$ & .023 \\
ICA terminus occlusion & $2.50(1.14-5.50)$ & .022 \\
Parenchymal hemorrhage & $5.06(1.30-19.7)$ & .020 \\
Lowest MAP (per 10-mm Hg drop & $1.28(1.01-1.62)$ & .043
\end{tabular}

below $100 \mathrm{~mm} \mathrm{Hg})^{b}$

${ }^{a}$ Variables with $P<.1$ in univariate analysis were entered simultaneously into a multivariate logistic regression model.

${ }^{b}$ Lowest intraprocedural MAP recorded prior to $\mathrm{mTICI} 2 \mathrm{~b} / 3$ reperfusion.
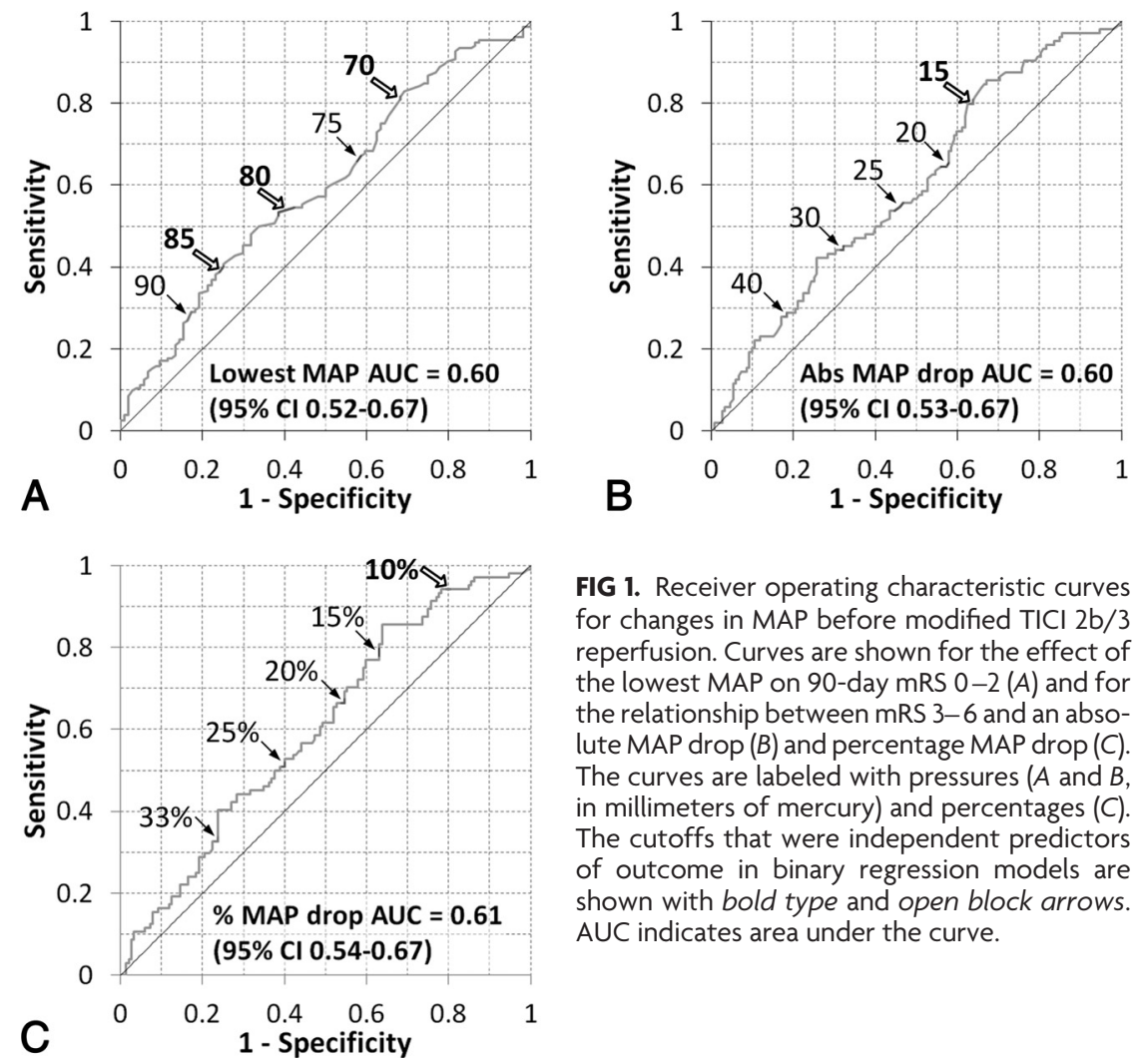

FIG 1. Receiver operating characteristic curves for changes in MAP before modified $\mathrm{TICl} 2 \mathrm{~b} / 3$ reperfusion. Curves are shown for the effect of the lowest MAP on 90-day mRS 0-2 (A) and for the relationship between $\mathrm{mRS} 3-6$ and an absolute MAP drop (B) and percentage MAP drop (C). The curves are labeled with pressures $(A$ and $B$, in millimeters of mercury) and percentages (C) The cutoffs that were independent predictors of outcome in binary regression models are shown with bold type and open block arrows. AUC indicates area under the curve. therefore appears that no particular sedative regimen can ensure tight hemodynamic control.

Not only are blood pressure drops common during MAC, but even modest drops in blood pressure can have a dramatic impact nologic outcome. This is perhaps best illustrated by our characteristic curves and multivariate mod MAP threshold. Instead, the lowest MAP showed a dose effect in which each 10-mm Hg drop below $100 \mathrm{~mm}$ Hg increased the odds of poor outcome. Although we consistently observed the benefit of keeping the MAP close to baseline, the critical MAP threshold may vary by the severity of the underlying stroke. Patients with an NIHSS score of $<15$ had high rates of good outcome across a wide range of MAPs, whereas the lowest MAP below $80 \mathrm{~mm} \mathrm{Hg}$ had a arge outcome effect for those with NIHSS scores of $>20$. For verity have poorer collaterals and, thus, are more dependent on blood pressure compared with patients with milder stroke severity.

Low blood pressure has been reported to be an independent risk factor for poor outcome in 2 mixed populations of MAC and patients under general anesthesia ${ }^{3,4}$ and 1 pure general anesthesia group. ${ }^{12}$ We found that this relationship holds true even in populations with no general anesthesia. What remains unknown, however, is whether intervention to correct hypotension can actually improve outcomes. One group recently reported their experience instituting hemodynamic parameters for their patients undergoing thrombectomy. ${ }^{15}$ They noted similar blood pressures in the groups with poor and favorable outcomes but that the cumulative dose of norepinephrine was independently associated with poor outcome. ${ }^{15}$ These data raise the possibility that vasopressors may improve peripheral blood pressures without improving blood flow to the ischemic penumbra. Future work might approach this question through a prospective, randomized trial evaluating strict blood pressure control by using vasopressors in comparison with usual anesthesia care. Until the results of such a trial are available, it seems reasonable to minimize hypotension during endovascular thrombectomies for acute ischemic stroke.

The present study has several limitations. Although larger than the previous studies, it remains retrospective in nature. As with all such studies, missing data may bias the results in unpredictable ways. For 


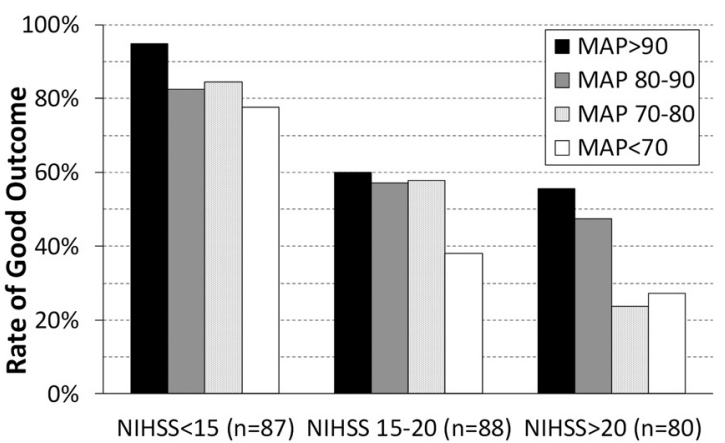

FIG 2. Rates of good neurologic outcome (90-day mRS of 0-2) by baseline NIHSS score and the lowest MAP measured before modified $\mathrm{TICl} 2 \mathrm{~b} / 3$ reperfusion. Subdividing the NIHSS groups into 4 MAP categories yielded subgroups of similar size $(n=18-26)$.

example, we did not collect prestroke mRS scores. The lowest blood pressures before reperfusion were captured from the anesthesia records as the lowest MAP rather than lowest systolic pressure. However, our rationale was that most blood pressure data were acquired by using noninvasive oscillometric cuff sphygmomanometers, which measure the MAP and derive systolic and diastolic pressures from an algorithm. ${ }^{7}$ Additionally, our choice of a study population with minimal confounders may limit the generalizability of our results to other patients undergoing thrombectomy, such as those with posterior circulation stroke or patients receiving general anesthesia. Nevertheless, the hemodynamic variables we identified may provide a useful way to control for the effect of hypotension in future studies on the effect of anesthesia on outcome.

\section{CONCLUSIONS}

Significant decreases in blood pressure occur during thrombectomies performed under MAC sedation. Our data support the hypothesis that hypotension influences outcome in patients not exposed to general anesthesia. Every 10-mm Hg drop in MAP below $100 \mathrm{~mm} \mathrm{Hg}$ before reperfusion increased the odds of poor outcome by an estimated $28 \%$ (95\% CI, 1\%-62\%). Even a $10 \%$ drop in MAP from the initial value seen in the angiography suite was a strong independent risk factor for poor outcome. These results suggest that clinicians should be just as vigilant in maintaining blood pressure for patients under MAC or conscious sedation as they are for those under general anesthesia.

Disclosures: Diogo C. Haussen-UNRELATED: Other Relationships: consultant for Codman (unpaid). Michael R. Frankel-UNRELATED: Grants/Grants Pending: Stryker, Comments: I am a coinvestigator on a clinical trial called DWI/PWI and CTP Assessment in the Triage of Wake-Up and Late Presenting Strokes Undergoing Neurointervention (DAWN), which tests whether thrombectomy using a device supplied by Stryker is effective in patients who are between 6 and 24 hours from symptom onset. I do not receive direct payment or direct salary support for this activity. I am just a coinvestigator. I was also a coinvestigator on the following clinical trials: A Randomized, Concurrent Controlled Trial to Assess the Safety and Effectiveness of the Separator 3D as a Component of the Penumbra System in the Revascularization of Large Vessel Occlusion in Acute Ischemic Stroke (Penumbra3D), Stent-Retriever Thrombectomy After Intravenous t-PA vs t-PA Alone in Stroke (SWIFT PRIME), and the Randomized, Concurrent Controlled Trial to Assess the Penumbra System's Safety and Effectiveness in the Treatment of Acute Stroke (THERAPY) (industrysponsored studies), which involved thrombectomy and used devices in patients who were included in the present article. I received no direct payment or direct salary support.* Raul G. Nogueira—RELATED: Fees for Participation in Review Activities such as Data Monitoring Boards, Statistical Analysis, Endpoint Committees, and the Like: Stryker Neurovascular, Medtronic, Penumbra, Comments: Stryker Neurovascular (Randomized Trial Evaluating Performance of the Trevo Retriever Versus the
Merci Retriever in Acute Ischemic Stroke [TREVO-2]; DAWN; Principal Investigator, no compensation); Medtronic (SOLITAIRE FR With the Intention for Thrombectomy [SWIFT] trial steering committee, modest compensation; [SWIFT-PRIME], Steering Committee, no compensation; Solitaire FR Thrombectomy for Acute Revascularization [STAR] trial Angiographic Core Lab, significant compensation); and Penumbra (A Randomized, Concurrent Controlled Trial to Assess the Safety and Effectiveness of the Separator 3D as a Component of the Penumbra System in the Revascularization of Large Vessel Occlusion in Acute Ischemic Stroke [Penumbra 3D], Executive Committee, no compensation); Editor-In-Chief of the journal Interventional Neurology (no compensation). *Money paid to the institution.

\section{REFERENCES}

1. Abou-Chebl A, Lin R, Hussain MS, et al. Conscious sedation versus general anesthesia during endovascular therapy for acute anterior circulation stroke: preliminary results from a retrospective, multicenter study. Stroke 2010;41:1175-79 CrossRef Medline

2. Abou-Chebl A, Zaidat OO, Castonguay AC, et al. North American SOLITAIRE Stent-Retriever Acute Stroke Registry: choice of anesthesia and outcomes. Stroke 2014;45:1396-401 CrossRef Medline

3. Davis MJ, Menon BK, Baghirzada LB, et al; Calgary Stroke Program. Anesthetic management and outcome in patients during endovascular therapy for acute stroke. Anesthesiology 2012;116:396-405 CrossRef Medline

4. Whalin MK, Lopian S, Wyatt K, et al. Dexmedetomidine: a safe alternative to general anesthesia for endovascular stroke treatment. J Neurointerv Surg 2014;6:270-75 CrossRef Medline

5. Zaidat OO, Yoo AJ, Khatri P, et al; Cerebral Angiographic Revascularization Grading (CARG) Collaborators, STIR Revascularization working group, STIR Thrombolysis in Cerebral Infarction (TICI) Task Force. Recommendations on angiographic revascularization grading standards for acute ischemic stroke: a consensus statement. Stroke 2013;44:2650-63 CrossRef Medline

6. Bruno A, Akinwuntan AE, Lin C, et al. Simplified modified Rankin Scale questionnaire: reproducibility over the telephone and validation with quality of life. Stroke 2011;42:2276-79 CrossRef Medline

7. Amoore JN. Oscillometric sphygmomanometers: a critical appraisal of current technology. Blood Press Monit 2012;17:80-88 CrossRef Medline

8. Talke PO, Sharma D, Heyer EJ, et al. Society for Neuroscience in Anesthesiology and Critical Care Expert Consensus Statement: anesthetic management of endovascular treatment for acute ischemic stroke $^{*}$ - endorsed by the Society of NeuroInterventional Surgery and the Neurocritical Care Society. J Neurosurg Anesthesiol 2014;26: 95-108 CrossRef Medline

9. Leonardi-Bee J, Bath PM, Phillips SJ, et al; IST Collaborative Group. Blood pressure and clinical outcomes in the International Stroke Trial. Stroke 2002;33:1315-20 CrossRef Medline

10. Castillo J, Leira R, Garcia MM, et al. Blood pressure decrease during the acute phase of ischemic stroke is associated with brain injury and poor stroke outcome. Stroke 2004;35:520-26 CrossRef Medline

11. Ritter MA, Kimmeyer P, Heuschmann PU, et al. Blood pressure threshold violations in the first $\mathbf{2 4}$ hours after admission for acute stroke: frequency, timing, predictors, and impact on clinical outcome. Stroke 2009;40:462-68 CrossRef Medline

12. Löwhagen Hendén P, Rentzos A, Karlsson JE, et al. Hypotension during endovascular treatment of ischemic stroke is a risk factor for poor neurological outcome. Stroke 2015;46:2678-80 CrossRef Medline

13. John S, Thebo U, Gomes J, et al. Intra-arterial therapy for acute ischemic stroke under general anesthesia versus monitored anesthesia care. Cerebrovasc Dis 2014;38:262-67 CrossRef Medline

14. Jagani M, Brinjikji W, Rabinstein AA, et al. Hemodynamics during anesthesia for intra-arterial therapy of acute ischemic stroke. $\mathrm{JNeu}$ rointerv Surg 2016;8:883-88 CrossRef Medline

15. Mundiyanapurath S, Stehr A, Wolf M, et al. Pulmonary and circulatory parameter guided anesthesia in patients with ischemic stroke undergoing endovascular recanalization. J Neurointerv Surg 2016;8: 335-41 CrossRef Medline 